\title{
SEQUENCES OF DIVIDED POWERS IN IRREDUCIBLE, COCOMMUTATIVE HOPF ALGEBRAS
}

\author{
BY \\ KENNETH NEWMAN $\left({ }^{1}\right)$
}

\begin{abstract}
In Hopf algebras with one grouplike element, M. E. Sweedler showed that over perfect fields, sequences of divided powers in cocommutative, irreducible Hopf algebras can be extended if certain "coheight" conditions are met. Here, we show that with a suitable generalization of "coheight", Sweedler's theorem is true over nonperfect fields. (We also point out, that in one case Sweedler's theorem was false, and additional conditions must be assumed.) In the same paper, Sweedler gave a structure theorem for irreducible, cocommutative Hopf algebras over perfect fields. We generalize this theorem in both the perfect and nonperfect cases. Specifically, in the nonperfect case, while a cocommutative, irreducible Hopf algebra does not, in general, satisfy the structure theorem, the sub-Hopf algebra, generated by all sequences of divided powers, does. Some additional properties of this sub-Hopf algebra are also given, including a universal property.
\end{abstract}

Some of the important definitions used in this paper are the following:

(1) If $H$ is a Hopf algebra and $0 \neq g \in H$, then $g$ is a grouplike if $\Delta g=g \otimes g$.

(2) If $h \in H$, then $h$ is a primitive if $\Delta h=h \otimes 1+1 \otimes h$. Note that the primitives of $H$ form a vector space. We will call it $P(H)$.

(3) A Hopf algebra $H$ will be called irreducible if every nontrivial subcoalgebra contains a fixed, nontrivial subcoalgebra, i.e. if $H$ is irreducible, it has a unique grouplike. (Note. For Hopf algebras, the terms "coconnected" and "irreducible" are synonymous.)

(4) A sequence of divided powers ${ }^{0} x=1,{ }^{1} x,{ }^{2} x, \ldots,{ }^{t} x$ is a set of elements in $H$ such that $\Delta^{n} x=\sum_{i=0}^{n}{ }^{i} x \otimes^{n-i} x$, for all $0 \leqq n \leqq t$.

Notation. Throughout the rest of this paper $k$ will be a field of char $p>0$ and $\bar{k}$ the perfect closure of $k$. $H$ will be an irreducible, cocommutative Hopf algebra over $k$, with augmentation $\varepsilon$, diagonalization $\Delta$, and identity $1 . \delta$ will be the map $[(\varepsilon-I) \otimes(\varepsilon-I)] \circ \Delta$, where $I$ is the identity map on $H$ and we identity $k$ with $k \cdot 1$. All tensor products will be over $k$, unless otherwise noted.

If $K$ is any extension field of $k$, we view $H \otimes K$ as a Hopf algebra over $K$, by letting $\Delta_{H \otimes K}: H \otimes K \rightarrow H \otimes K \otimes_{K} H \otimes K \approx H \otimes H \otimes K$ equal $\Delta \otimes I_{K}$ and by

Received by the editors September 2, 1970.

AMS 1970 subject classifications. Primary 16A24.

Key words and phrases. Coheight, irreducible Hopf algebra, sequence of divided powers.

(1) This paper is similar to a portion of my doctoral dissertation written under the direction of Professor Stephen U. Chase. I would also like to thank Professor Moss E. Sweedler for his many valuable suggestions.

Copyright (C) 1972, American Mathematical Society 
letting $\varepsilon_{H \otimes K}=\varepsilon \otimes I_{K}$. Further, if $h \otimes \alpha, h^{\prime} \otimes \alpha^{\prime} \in H \otimes K$, then $(h \otimes \alpha)\left(h^{\prime} \otimes \alpha^{\prime}\right)$ $=h h^{\prime} \otimes \alpha \alpha^{\prime}$.

Lemma 1. Let ${ }^{0} x,{ }^{1} x, \ldots,{ }^{n} x$ be a sequence of divided powers in $H$. Let $K$ be any extension field of $k$. Then ${ }^{0} x,{ }^{1} x, \ldots,{ }^{n} x$ can be extended in $H$ if and only if the sequence of divided powers ${ }^{0} x \otimes 1,{ }^{1} x \otimes 1, \ldots,{ }^{n} x \otimes 1$ cai be extended in $H \otimes K$.

Proof. $\Rightarrow$ obvious.

$\Leftarrow$ We want to find a $y \in H$ such that $\delta(y)=\sum_{i=1}^{n}{ }^{i} x \otimes{ }^{n+1-i} x$. Pick $z$ $=\sum_{i \in I} y_{i} \otimes \lambda_{i}$ in $H \otimes K$ such that $(\delta \otimes I)(z)=\sum_{i=1}^{n}{ }^{i} x \otimes^{n+1-i} x \otimes 1$. Pick a maximal subset $J \subset I$ such that $\left\{\delta\left(y_{i}\right)\right\}_{i \in J}$ will be an independent set in $H \otimes H$. Then clearly we can find $\left\{\tau_{i}\right\}_{i \in J}$ in $K$ such that $(\delta \otimes I)\left(\sum_{i \in J} y_{i} \otimes \tau_{i}\right)=\sum_{i=1}^{n}{ }^{i} x \otimes{ }^{n+1-i} x \otimes 1$. Since the $\left\{\delta\left(y_{i}\right) \otimes 1\right\}_{i \in J}$ are independent over $K$ and

$$
\sum_{i \in J} \delta\left(y_{i}\right) \otimes \tau_{i}=(\delta \otimes I)\left(\sum_{i \in J} y_{i} \otimes \tau_{i}\right)
$$

is in $H \otimes H \otimes k$, by uniqueness of linear combinations of independent elements, the $\left\{\tau_{i}\right\}_{i \in J}$ must be in $k$. Therefore, $\sum_{i \in J} \tau_{i} y_{i}$ is the desired extension.

Definitions. (1) Let $k^{1 / p}=\left\{\alpha \in \bar{k} \mid \alpha^{p} \in k\right\}$. Let $\left\{x_{i}\right\}$ be a basis of $H$. Define

$$
v: H^{[p]} \equiv \underbrace{H \otimes H \otimes \cdots \otimes H}_{p \text { times }} \rightarrow H \otimes k^{1 / p}
$$

via

(a) $x_{i}^{[p]} \rightarrow x_{i} \otimes 1$

(b) all other basis elements $\rightarrow 0$.

Extend by $1 / p$-linearity.

It is shown in [1, Theorem 4.1.1 and Corollary 4.1.2] that on the p-fold symmetric tensors in $H^{[p]}, v$ is independent of basis. Throughout this paper, the domain of $v$ will always be the $p$-fold symmetric tensors.

(2) Define $V: H \rightarrow H \otimes k^{1 / p}$ as the composite $H \stackrel{\Delta_{p-1}}{\longrightarrow} H^{[p]} \stackrel{v}{\rightarrow} H \otimes k^{1 / p}$. Note that $V$ is $1 / p$-linear.

(3) Let $V^{n}(H)=V(\cdots(V(V(V(H) \cap H) \cap H) \cdots) \cap H)$ (n $V^{\prime}$ 's and $n H$ 's).

(4) Let $V^{\infty}(H)=\bigcap_{i=1}^{\infty} V^{i}(H)$.

(5) $x \in H$ has coheight $n$ if $x \in V^{n}(H)$.

It is not difficult to see that if $k$ is perfect, the definitions of " $V$ " and "coheight" given here correspond to those of [4].

(6) For $0<e$, let $\|e\| \in Z$ be defined by $p^{\|e\|} \leqq e<p^{\|e\|+1}$.

In [4] the next theorem is proved with the assumption that $k$ is perfect. Here we drop that assumption.

THEOREM 2. Assume we are given a sequence of divided powers ${ }^{0} x,{ }^{1} x, \ldots,{ }^{t-1} x$ satisfying: ${ }^{e} x$ has coheight $n-\|e\|$ for $1 \leqq e<t$ and $t<p^{n+1}$; then there is $a^{t} x$ of coheight $n-\|t\|$ such that ${ }^{0} x,{ }^{1} x, \ldots,{ }^{t} x$ is a sequence of divided powers. 
Note. I point out in [2, pp. 15-27] that it is only necessary to assume that ${ }^{e} x$ has coheight $n-\|t\|+i$ if $e \leqq t / p^{i}$. This weakened hypothesis has the virtue of being necessary. (This is not hard to see.)

Proof. The definition of $V^{n}$ implies that $V^{n-\|e\|}(H)=V^{\|t\|-\|e\|}\left(V^{n-\|t\|}(H) \cap H\right)$. Therefore ${ }^{e} x$ has coheight $\|t\|-\|e\|$ in $V^{n-\|e\|}(H)$. It follows that ${ }^{e} x \otimes 1$ has coheight $\|t\|-\|e\|$ in $\left(V^{n-\|t\|}(H) \cap H\right) \otimes \bar{k}$. Since $\bar{k}$ is perfect we can apply Lemma 7, p. 522 of [4], and find an extension of ${ }^{0} x \otimes 1,{ }^{1} x \otimes 1, \ldots,{ }^{t-1} x \otimes 1$ in $\left(V^{n-\|t\|}(H) \cap H\right) \otimes \bar{k}$. Lemma 1 now implies that there exists an extension in $V^{n-\|t\|}(H) \cap H$. By definition this extension will have coheight $n-\|t\|$ in $H$. Q.E.D.

Sublemma 3. Let ${ }^{0} x,{ }^{1} x, \ldots,{ }^{t} x$ be a sequence of divided powers. Then $V\left({ }^{i} x\right)=0$ if $p \nmid i$ and $V\left({ }^{i} x\right)={ }^{i / p} x$ if $p \mid i$.

Proof. Proposition 4.1 .9 of [1].

THEOREM 4. Let ${ }^{1} x \in P(H)$.

(a) ${ }^{1} x$ has coheight $n$ ( $n$ finite) if and only if there exists a sequence of divided powers ${ }^{0} x,{ }^{1} x, \ldots,{ }^{p^{n+1}-1} x$.

(b) ${ }^{1} x$ has coheight $\infty$ if and only if for any $t$ there exists a sequence of divided powers ${ }^{0} x,{ }^{1} x, \ldots,{ }^{t} x$.

(c) If ${ }^{1} x$ has coheight $\infty$ and $V \mid V^{\infty}(H) \rightarrow V^{\infty}(H) \otimes k^{1 / p}$ is onto, then there will exist a sequence of divided powers of infinite length over ${ }^{1} x$.

(See Corollary 13, below.)

Proof. (a) $\Rightarrow$ Assume inductively that we have found ${ }^{0} x,{ }^{1} x, \ldots,{ }^{t} x\left(t<p^{n+1}-1\right)$ such that the coheight of ${ }^{e} x$ is $n-\|e\|$ for all $1 \leqq e \leqq t$. Then Theorem 2 implies we can find ${ }^{t+1} x$ of coheight $n-\|t+1\|$ extending the sequence. Q.E.D.

(a) $\Leftarrow$ The sublemma implies $V^{i}\left({ }^{p^{n}} x\right)={ }^{p^{n-1}} x$. Therefore ${ }^{1} x \in V^{n}(H)$.

(b) $\Rightarrow$ If ${ }^{1} x$ has coheight $\infty$ then, a fortiori, it has coheight $n$ for any finite $n$. Apply part (a).

(b) $\Leftarrow$ Clear.

(c) Proposition 4.1.6 of [1] implies that $V(H)$ is a Hopf algebra over $k^{1 / p}$. It is clear that $V(H) \cap H$ will be a Hopf algebra over $k$, and by induction we can see that $V^{\infty}(H) \cap H$ will also be a Hopf algebra over $k$. Start constructing a sequence of divided powers over ${ }^{1} x$ in $V^{\infty}(H) \cap H$. Since $V \mid V^{\infty}(H) \rightarrow V^{\infty} \otimes k^{1 / p}$ is onto, each element in the sequence will have infinite coheight in $V^{\infty}(H) \cap H$. Therefore, the sequence can be extended indefinitely.

Note. In [4], Sweedler incorrectly states in Theorem 2, p. 521, that a primitive will have an infinite sequence of divided powers over it, if its coheight is $\infty$, i.e. he does not assume $V \mid V^{\infty}(H)$ is onto. The following example shows that his statement is false.

EXAMPLE 1. Let

$$
H=\frac{k\left[X ; X_{11} ; X_{21}, X_{22} ; X_{31}, X_{32}, X_{33} ; \ldots\right]}{\left(X^{p} ; X_{11}^{p} ; X_{21}^{p}, X_{22}^{p} ; X_{31}^{p}, X_{32}^{p}, X_{33}^{p} ; \ldots\right)}
$$


with $\Delta 1=1 \otimes 1, \Delta X=1 \otimes X+X \otimes 1$ and $\Delta X_{i j}=\sum_{\alpha=-1}^{j} X_{i \alpha} \otimes X_{i, j-\alpha-1}$. (Here $X_{i, 0}=X$ and $X_{i,-1}=1$ for all $i$.) Also let $\varepsilon(X)=\varepsilon\left(X_{i j}\right)=0$ and $\varepsilon(1)=1$. It is straightforward to check that $\Delta$ is coassociative and that the coalgebra generated by any element contains the identity. Therefore $H$ is an irreducible bialgebra, which implies that it is a Hopf algebra [3, Theorem 9.2.2, p. 193]. Note that $1, X, X_{i 1}, X_{i 2}$, $\ldots, X_{i i}$ is a sequence of divided powers for any $i$, which implies via Sublemma 3 that $V\left(X_{i j}\right)=0$ if $p \nmid j+1$ and $V\left(X_{i j}\right)=X_{i m}(m=(j+1 / p)-1)$ if $p \mid j+1$. Therefore, $X \in V^{\infty}(H)$, but any element in $V^{-1}(X)$ has finite coheight. This implies that any $p$ th divided power of $X$ has finite coheight, i.e. there does not exist an infinite sequence of divided powers over $X$.

Lemma 5. Let $K$ be any extension field of $k$. Then $P(H) \otimes K \approx P(H \otimes K)$.

Proof. It is clear that $\delta(x)=0$ and $\varepsilon(x)=0 \Leftrightarrow x \in P(H)$. Therefore

$$
0 \longrightarrow P(H) \longrightarrow H^{+} \stackrel{\delta}{\longrightarrow} H \otimes H
$$

is an exact sequence $\left(H^{+}=\{x \in H \mid \varepsilon(x)=0\}\right)$. Since $-\otimes K$ is an exact functor, we see that $P(H) \otimes K$ and $P(H \otimes K)$ are both the kernel of the same map and, therefore, are isomorphic.

LEMMA 6. Let $K$ be any extension field of $k$. Assume there exists a basis $\left\{x_{i}\right\}_{i \in J}$ of $H$ such that $V^{n}\left(x_{i}\right) \in H$ for all $i \in J$ (fixed $\left.n\right)$. Let $L_{n} \subset H$ be the vector space of primitives of coheight $n$. Then $L_{n} \otimes K$ is the space of primitives of coheight $n$ in $H \otimes K$.

Now assume the hypothesis is true for all $n$. Let $L_{\infty} \subset H$ be the vector space of primitives of coheight $\infty$. Then $L_{\infty} \otimes K$ is the vector space of primitives of coheight $\infty$ in $H \otimes K$.

Proof. It is clear that if $y \in V^{n}(H) \cap H$ then $y \otimes 1 \in V^{n}(H \otimes K)$, i.e. each element of $L_{n} \otimes K$ has coheight $n$ in $H \otimes K$. Let $M$ be a subspace of primitives complementary to $L_{n}$ in $P(H)$. Since $P(H \otimes K) \approx P(H) \otimes K \approx(M \otimes K) \oplus\left(L_{n} \otimes K\right)$ we only need show that every element of $M \otimes K$ has coheight less than $n$. Assume there exists $m=\sum m_{j} \otimes \alpha_{j} \in M \otimes K$ such that $m$ has coheight $n$. Pick $x \in H \otimes K$ such that $V^{n}(x)=m$. Write $x$ as $\sum x_{j} \otimes \beta_{j}$, where the $x_{j}$ are in the basis of $H$ picked in the hypothesis and the $\beta_{j} \in K$. Since $V^{n}$ is $1 / p^{n}$-linear, $V^{n}\left(\sum x_{j} \otimes \beta_{j}\right)$ $=\sum \beta_{j}^{1 / p^{n}}\left(V^{n}\left(x_{j}\right) \otimes 1\right)$, where, by hypothesis, $V^{n}\left(x_{j}\right) \in H$. Let $W$ be the subspace of $H$ spanned by the $V^{n}\left(x_{j}\right)$. Then $(W \otimes \bar{k}) \cap(M \otimes \bar{k}) \neq 0 \Rightarrow W \cap M \neq 0 \Rightarrow$ there exists an element in $M$ of coheight $n$. This contradicts the assumption that $L_{n}$ $=$ space of primitives of coheight $n$.

If the hypothesis is true for all $n$, then the space of primitives of infinite coheight in $H \otimes K$ equals $\bigcap_{i=1}^{\infty}\left(L_{i} \otimes K\right)=\left(\bigcap_{i=1}^{\infty} L_{i}\right) \otimes K=L_{\infty} \otimes K$.

Sublemma. If ${ }^{0} x,{ }^{1} x, \ldots,{ }^{n} x$ and ${ }^{0} y,{ }^{1} y, \ldots,{ }^{n} y$ are two sequences of divided powers, then $\left\{\sum_{j=0}^{i}{ }^{j} x \cdot{ }^{i-j} y\right\}_{i=0}^{n}$ is a sequence of divided powers. 
Proof. Let $C$ be the coalgebra with basis consisting of the sequence of divided powers $1,{ }^{1} u, \ldots,{ }^{n} u$. Then we have coalgebra maps $f: C \rightarrow H, g: C \rightarrow H$ defined by $f\left({ }^{i} u\right)={ }^{i} x, g\left({ }^{i} u\right)={ }^{i} y$. The coalgebra map $f * g: C \rightarrow H$, defined to be the composite

$$
C \stackrel{\Delta_{C}}{\longrightarrow} C \otimes C \stackrel{f \otimes g}{\longrightarrow} H \otimes H \stackrel{\text { mult }}{\longrightarrow} H,
$$

takes ${ }^{i} u$ to $\sum_{j=0}^{i}{ }^{i} x \cdot{ }^{i-j} y$. Consequently $\sum_{j=0}^{i}{ }^{j} x \cdot{ }^{i-j} y, i=0, \ldots, n$, is a sequence of divided powers in $H$.

Lemma 7 (B. PAReIGIS). For every $l$ in $P(H)$ pick a sequence of divided powers of length $s$ over it (or of length $p^{t+1}-1$ if $t=$ maximal coheight of $l$ and $p^{t+1}-1<s$ ). Call it ${ }^{0} l,{ }^{1} l,{ }^{2} l, \ldots,{ }^{s} l$. Then any sequence of divided powers of length $s$ in $H$ can be written in the form:

$$
\left\{\sum_{e_{1}+2 e_{2}+\cdots+s e_{s}=n} e_{1} l_{1} e_{2} l_{2} \ldots e_{s} l_{s}\right\}_{(n=0,1, \ldots, s)}
$$

Proof. Let ${ }^{0} l_{1},{ }^{1} l_{1},{ }^{2} \lambda_{1},{ }^{3} \lambda_{1}, \ldots,{ }^{s} \lambda_{1}$ be any sequence of divided powers of length $s$ over $l_{1}$. Since ${ }^{2} \lambda_{1}$ and ${ }^{2} l_{1}\left({ }^{2} l_{1}\right.$ picked in hypothesis) extend the same sequence of divided powers, ${ }^{2} l_{1}-{ }^{2} \lambda_{1}$ is a primitive, say ${ }^{1} l_{2}$. By the sublemma,

$$
\left\{{ }^{n} \lambda_{2} \equiv \sum_{e_{1}+2 e_{2}=n} e_{1} l_{1} e_{2} l_{2}\right\}_{(n=0,1, \ldots, s)}
$$

will be a sequence of divided powers of length $s$ starting with $1,{ }^{1} l_{1},{ }^{2} \lambda_{1}$. (We are " adding" the sequences $1,{ }^{1} l_{1},{ }^{2} l_{1},{ }^{3} l_{1}, \ldots$ and $1,0,{ }^{1} l_{2}, 0,{ }^{2} l_{2}, \ldots$ ) Again, the third term in this sequence will differ from ${ }^{3} \lambda_{1}$ by a primitive, say ${ }^{1} l_{3}$, and we can write (using an obvious generalization of the sublemma):

$$
\left\{{ }^{n} \lambda_{3} \equiv \sum_{e_{1}+2 e_{2}+3 e_{3}=n} e_{1} l_{1} e_{2} l_{2} e_{3} l_{3}\right\}_{(n=0,1, \ldots, s)}
$$

a sequence of divided powers starting with $1,{ }^{1} l_{1},{ }^{2} \lambda_{1},{ }^{3} \lambda_{1}$. Continue.

Note. The sequence over each ${ }^{1} l_{i}$ will always be as long as necessary, i.e. its

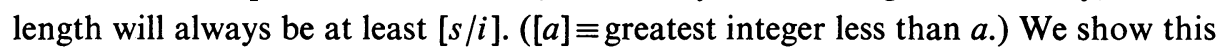
by proving that the coheight of ${ }^{j} l_{i}$ is $\|s / i\|-\|j\|$, so in particular the coheight of ${ }^{1} l_{i}$ is $\|s / i\|$, which implies by Theorem 4 that the sequence over ${ }^{1} l_{i}$ has length at least $p^{\|s / i\|+1}-1 \geqq[s / i]$. Assume inductively that coheight ${ }^{j} l_{i}=\|s / i\|-\|j\|$ for all $i<m$. Then since

(1) $\|s / i\|-\|j\| \geqq\|s / m\|$ if $i j \leqq m \Rightarrow$ coheight of ${ }^{j} l_{i}$ is $\geqq\|s / m\|$ if $i j \leqq m, i<m$,

(2) coheight ${ }^{m} \lambda_{1}$ is $\|s / m\|$,

(3) $V^{\|s / m\|}(H)$ is a Hopf algebra, and

(4) ${ }^{1} l_{m}={ }^{m} \lambda_{1}-\sum_{e_{1}+2 e_{2}+\cdots+(m-1) e_{m-1}=m} e_{1} l_{1} e_{2} l_{2} \ldots{ }^{e_{m-1}} l_{m-1}$, we see that ${ }^{1} l_{m}$ has coheight $\|s / m\|$. To complete the induction, we note that if the sequence picked in the hypothesis over ${ }^{1} l_{m}$ has length $s$, then ${ }^{j} l_{m}$ has coheight $\|s / j\|$ and since $\|s / m\|-\|j\| \leqq\|s / j\|$, a fortiori, has coheight $\|s / m\|-\|j\|$. If the chosen sequence 
were shorter, then by hypothesis, it is of maximal length over ${ }^{1} l_{m} \Rightarrow{ }^{j} l_{m}$ has coheight $\|s / m\|-\|j\|$. (See proof of Theorem 4(a).) Q.E.D.

On p. 518 of [4], Sweedler shows that if $E \equiv I-\varepsilon, \delta^{n} \equiv E^{[n+1]} \Delta_{n}$, and $H_{n}$ $\equiv \operatorname{ker} \delta^{n}(H)$, then $\delta^{n-1}\left(H_{n}\right) \subset T$ where $T$ represents the tensor algebra on $P(H)$. Furthermore, if we let $V$ be a subspace of $H$ and let $V_{i} \equiv V \cap H_{i}$ and $T_{V}$ $\equiv V_{0} \oplus\left(\oplus_{1}^{\infty} \delta^{i-1}\left(V_{i}\right)\right)$, we have the following

Lemma 8. If $V \subset W$ are subspaces of $H$, then $V=W$ if and only if $T_{V}=T_{W}$.

Definitions. (1) Let $G$ be an ordered set (finite or infinite) and for $\gamma \in G$ let $0 \leqq e_{\gamma} \in Z$. The vector $\left(e_{\gamma}\right)_{\gamma \in G}$ will be denoted $e$. Let $|e| \equiv \sum e_{\gamma}$.

(2) Let $W$ be a vector space with basis $\left\{w_{\gamma}\right\}_{\gamma \in G}$. For $e$ such that $\infty>|e|>0$, let $S W^{e}$ denote the symmetric tensor generated by $\left.\cdots \otimes w_{\gamma}^{[e} \gamma\right] \otimes \cdots$ If $|e|=0$, let $S W^{e}=1$.

(3) A Sweedler basis of $H$ will be a basis of $P(H),\left\{l_{\gamma}\right\}_{\gamma \in G_{0}}$ such that there exists $G_{0} \supset G_{1} \supset \ldots \supset G_{\infty}$ such that $\left\{l_{\gamma}\right\}_{\gamma \in G_{i}}$ is a basis of the primitives of coheight $n$.

(4) Denote the maximum coheight of ${ }^{1} l_{\gamma}$ by $(\gamma)$.

THEOREM 9. Assume $H$ has a Sweedler basis $\left\{l_{\gamma}\right\}_{\gamma \in G_{0}}$ with $G_{0}$ ordered. If $\gamma \in G_{i}$, consider $\left\{{ }^{i} l_{\gamma}\right\}_{i=0}^{p(\gamma)+1-1}$ where ${ }^{i} l_{\gamma}$ is the ith element in some sequence of divided powers over ${ }^{1} l_{\gamma}$. (The ${ }^{i} l_{\gamma}$ do not necessarily have to be in the same sequence.) The ${ }^{i} l_{\gamma}$ exist by Theorem 4. Then there exists a sub-Hopf algebra $H^{\prime} \subset H$ with basis

$$
\left\{e_{1} l_{\gamma_{1}} \ldots e_{m} l_{\gamma_{m}} \equiv \prod^{e} l\right\} \quad\left(\gamma_{1}<\gamma_{2}<\cdots<\gamma_{m} \text { and } e_{\gamma}<p^{(\gamma)+1}\right) .
$$

$H^{\prime}$ is independent of which Sweedler basis was picked, or which sequences of divided powers the ${ }^{i} l_{\gamma}$ were picked from. In particular, any element of any sequence of divided powers in $H$ will be in $H^{\prime}$.

Note. We do not really need that $\left(\gamma_{1}<\gamma_{2}<\cdots<\gamma_{m}\right)$ but just that there is one monomial corresponding to each finite subset of elements of sequences of divided powers, no two subscripts equal. In [2, Theorem 1.1.14, pp. 24-25], I give some further generalizations.

Note. In [4] Sweedler has a corresponding theorem for Hopf algebras over perfect fields. However, there he assumes that, for $\gamma \in G_{i}, 1,{ }^{1} l_{\gamma},{ }^{2} l_{\gamma},{ }^{3} l_{\gamma}, \ldots$ form a sequence of divided powers. As I pointed out in Example 1, if $i=\infty$, such a sequence is not always possible to construct, and, therefore, the change in the hypothesis is significant.

EXAMPLE 2. Let $k$ be a nonperfect field of char $p>0$ and assume $\alpha \in k$ but $\alpha \notin k^{p}$. Let $H=k[X, Y, Z] /\left(X^{p}, Y^{p}, Z^{p}\right)$ and give $H$ a Hopf algebra structure by letting $\Delta X=X \otimes 1+1 \otimes X, \Delta Y=Y \otimes 1+1 \otimes Y$, and

$$
\Delta Z=Z \otimes 1+1 \otimes Z+\sum_{i=1}^{p-1} \frac{1}{i !} \frac{1}{(p-i) !} X^{i} \otimes X^{p-i}+\alpha \sum_{i=1}^{p-1} \frac{1}{i !} \frac{1}{(p-i) !} Y^{i} \otimes Y^{p-i}
$$


and $\varepsilon(X)=\varepsilon(Y)=\varepsilon(Z)=0$. It is straightforward to compute that $\delta^{p-1}(Z)$ $=X^{[p]}+\alpha Y^{[p]}$ and this implies that $V(Z)=X \otimes 1+Y \otimes \alpha^{1 / p} \in H \otimes k^{1 / p}$. Further, since $V$ is a $1 / p$-linear map $V(a Z)=X \otimes a^{1 / p}+Y \otimes \alpha^{1 / p} a^{1 / p} \notin H \otimes k$ for all $a \in k$. Therefore, since $V(X)=V(Y)=0$ we obtain that $V(H) \cap H=k$. Consequently, all the elements of $H^{+}$, and in particular, the primitives, have coheight 0 and a Sweedler basis of $H$ will be $G_{i}=\varnothing, i>0$, and $G_{0}=\{X, Y\}$. (That the primitives are a 2-dimensional subspace can be computed, but it will be evident from the theorem and the explanation below.) Now

$$
{ }^{i} X \equiv \frac{X^{i}}{i !} \quad(i=0,1, \ldots, p-1) \quad \text { and } \quad{ }^{i} Y \equiv \frac{Y^{i}}{i !} \quad(i=0,1, \ldots, p-1)
$$

will be a $p-1$ length sequence of divided powers over $X$ and $Y$, respectively $\Rightarrow$ in this case, $H^{\prime}=k[X, Y] /\left(X^{p}, Y^{p}\right)$.

Now consider $H \otimes k^{1 / p}$. Here $X \otimes 1+Y \otimes \alpha^{1 / p}$ is a primitive of coheight 1 and, therefore, a Sweedler basis of $H \otimes k^{1 / p}$ will be $G_{i}=\varnothing(i>1), G_{1}=\left\{X \otimes 1+Y \otimes \alpha^{1 / p}\right\}$, $G_{0}=\left\{X \otimes 1+Y \otimes \alpha^{1 / p}, X \otimes 1\right\}$. (Since $P\left(H \otimes k^{1 / p}\right)$ has dimension 2 and $X \otimes 1 \in P\left(H \otimes k^{1 / p}\right)$ has coheight 0 , the subspace of primitives of coheight 1 is one-dimensional. $\operatorname{dim} P(H)=\operatorname{dim} P\left(H \otimes k^{1 / p}\right)$ by Lemma 5.) Since each element of $\left\{(1 / i !)\left(X \otimes 1+Y \otimes \alpha^{1 / p}\right)^{i}\right\}(i=0,1, \ldots, p-1)($ a $p-1$ sequence of divided powers over $\left.X \otimes 1+Y \otimes \alpha^{1 / p}\right)$ has coheight 1 , we can extend to a $p^{2}-1$ sequence of divided powers. (For instance, a $p$ th power will be

$$
Z+\sum_{i=1}^{p-1} \frac{1}{i !} \frac{1}{(p-i) !}(X \otimes 1)^{i}(Y \otimes \alpha)^{p-i} .
$$

We know by Theorem 4 that we can extend to a sequence of length $p^{2}-1$.) The theorem implies that monomials of the form

$\left\{(X \otimes 1)^{i j}\left(X \otimes 1+Y \otimes \alpha^{1 / p}\right) \mid i=0,1, \ldots, p-1\right.$ and $j=0,1, \ldots, p^{2}-1 ;$

$$
\left.{ }^{j}\left(X \otimes 1+Y \otimes \alpha^{1 / p}\right) \equiv j \text { th divided power of } X \otimes 1+Y \otimes \alpha^{1 / p}\right\}
$$

will be a basis of $H \otimes k^{1 / p}$.

Proof of Theorem. Let $H^{\prime} \equiv$ the Hopf algebra generated by the monomials picked in the hypothesis. Note that the coalgebra generated by these monomials is spanned by a subset of all monomials consisting of elements of sequences of divided powers since

$$
\Delta\left(e_{1} l_{1} e_{2} l_{2} \ldots e_{m} l_{m}\right)=\sum_{\left(i_{1}, i_{2}, \ldots, i_{m}\right)=(0,0, \ldots, 0)}^{\left(e_{1}, e_{2}, \ldots, e_{m}\right)} i_{1} l_{1}^{i_{2}} l_{2} \ldots i_{m} l_{m} \otimes e_{1}-i_{1} l_{1} e_{2}-i_{2} l_{2} \ldots e_{m}-i_{m} l_{m} .
$$

Sublemma 3, together with the fact that $V$ commutes with multiplication [1, Propositions 4.1.9 and 3.1.6(d)], implies that $V^{n}$ (for any $n$ ) applied to any element in this spanning set is in $H^{\prime} \otimes k^{1 / p} \cap H=H^{\prime}$. Therefore, again using that $V$ commutes with multiplication, there is a basis $\left\{x_{i}\right\}_{i \in J}$ of $H^{\prime}$ such that $V^{n}\left(x_{i}\right) \in H^{\prime}$ for all $i$ and $n$. 
Now consider $H^{\prime} \otimes \bar{k}$. Lemma 6 implies that since $\left\{l_{\gamma}\right\}_{\gamma \in G_{0}}$ is a Sweedler basis of $H^{\prime},\left\{l_{y} \otimes 1\right\}_{\gamma \in G_{0}}$ is a Sweedler basis of $H^{\prime} \otimes \bar{k}$. We first want to show that the image of the monomials picked in the hypothesis, under the natural injection of $H^{\prime}$ into $H^{\prime} \otimes \bar{k}$, span $H^{\prime} \otimes \bar{k}$. Lemma 6 of [4] says, in essence, that if $h \in H^{\prime} \otimes \vec{k}$ and $\delta^{m}(h)=0$, then $\delta^{m-1}(h)$ can be written in the form $\sum_{i} \alpha_{i} S(l \otimes 1)^{e_{i}}$ with $\alpha_{i} \in \bar{k}$, the $l \otimes 1$ in the Sweedler basis of $H^{\prime} \otimes \bar{k}$ and $e_{\gamma_{i}}<p^{(\gamma)+1}$ for all $i$. Now it is not difficult to see that if $\prod^{e} l$ is one of the monomials picked in the hypothesis and $|e|=n$, then $\delta^{n-1}\left(\prod^{e} l\right)=S l^{e}$. Therefore, for any $h \in H^{\prime} \otimes \bar{k}$ we can find $M$ (a linear combination of the monomials picked in the hypothesis) such that $\delta^{m-1}(h)$ $=\delta^{m-1}(M \otimes 1)$. (If $\delta^{m-1}(h)=\sum_{i} \alpha_{i} S(l \otimes 1)^{e_{i}}$, let $M=\sum_{i} \alpha_{i}\left(\Pi{ }^{\left.e_{i} l\right)}\right)$.) Lemma 8 now implies that the space spanned by these monomials (thought of as elements of $H$ ) is $H^{\prime}$.

We now show independence. Assume there exists $\left\{a_{i}\right\} \in k-\{0\}$ and distinct polynomials $y_{i} \equiv \prod_{i} e_{i} l$ such that $x \equiv \sum_{i} a_{i} y_{i}=0$. Pick the largest $n$ such that $\delta^{n}$ applied to some $y_{i} \neq 0$ (i.e. $n$ will be the largest $\left|e_{i}\right|-1$ ). Then $0=\delta^{n}(x)=\sum_{J} a_{i} S l^{e_{i}}$ where $J=\left\{i|| e_{i} \mid=n+1\right\}$. Note however that the $S l^{e_{i}}$ 's are independent, since the $l_{\gamma}$ 's are independent. This is a contradiction.

Now we will show that any element of any sequence of divided powers is in $H^{\prime}$. Let ${ }^{n} l \in H$ be the $n$th term in some sequence of divided powers. Now pick sequences of divided powers over every primitive in $H$ as per Lemma 7. Since every primitive has the same coheight in $H$ and $H^{\prime}$, we can assume that these sequences are in $H^{\prime}$. (See Lemma 4.) Now Lemma 7 says that ${ }^{n} l$ is a sum and product of elements in $H^{\prime} \Rightarrow{ }^{n} l$ is in $H^{\prime}$.

Now finally by symmetry, we can conclude that the elements of the sequences of divided powers we picked in the hypothesis will be in the Hopf algebra generated by any alternative set of elements of sequences of divided powers.

COROLLARY 10. If $G$ is a Hopf algebra over $k$ generated by sequences of divided powers and if $\varphi: G \rightarrow H$ is a Hopf algebra map, then $\varphi$ factors uniquely through $H^{\prime} \hookrightarrow H$, i.e. the assignment of $H$ to $H^{\prime}$ is functorial and this functor is a right adjoint to the inclusion functor.

Proof. Since the image of a sequence of divided powers is a sequence of divided powers, the image of $\varphi$ must lie in $H^{\prime}$.

REMARK 11. By Lemma 6 of [4] (the one referred to in the proof of Theorem 9), it is clear that over perfect fields $H^{\prime}=H$. (This is one of the important results of [4].)

REMARK 12. Over a perfect field, even if $H$ does not have a Sweedler basis, $H$ is spanned by monomials whose factors are elements of sequences of divided powers. In particular, $H$ is generated as an algebra by elements of sequences of divided powers.

Proof. For every $n$ (finite or infinite), pick a basis $\left\{{ }^{1} l_{i n}\right\}$ of primitives of coheight $n$. For each ${ }^{1} l_{i n}$ pick a sequence of elements $1,{ }^{1} l_{i n},{ }^{2} l_{i n}, \ldots,{ }^{p^{n+1}-1} l_{i n}$, such that ${ }^{j} l_{i n}$ is the $j$ th term of some sequence of divided powers over ${ }^{1} l_{i n}$. The monomials 
generated by all these elements will now span $H$. This is shown using Lemma 6 of [4], as in the proof of Theorem 9.

Corollary 13. If $P(H)$ is finite dimensional, then $V \mid V^{\infty}(H) \rightarrow V^{\infty}(H) \otimes k^{1 / p}$ is onto.

(This applies to the hypothesis of Theorem 4(c).)

Proof. (In [5], Sweedler gives a proof of this corollary using a theorem of R. Heyneman that $\operatorname{dim} P(H)<\infty \Rightarrow H$ is conoetherian, i.e. every decreasing sequence of subcoalgebras in $H$ stabilizes. The proof given here is more elementary.)

We first show that if ${ }^{1} x \in P(H)$ has infinite coheight, there exists an infinite sequence of divided powers over ${ }^{1} x$. Assume not. Let ${ }^{0} x,{ }^{1} x, \ldots,{ }^{t} x$ be a sequence of divided powers in $V^{\infty}(H)$ that cannot be extended in $V^{\infty}(H)$. Theorem 2 implies that for any finite $n$, there exists an extension of this sequence of coheight $n$. Therefore, we can find an infinite set of elements $\left(y_{1}, y_{2}, \ldots\right)$ such that the maximal coheight of $y_{i}<$ maximal coheight of $y_{i+1}$ and such that each $y_{i}$ is an extension of ${ }^{0} x,{ }^{1} x, \ldots,{ }^{t} x$. Since $y_{i+1}$ and $y_{i}$ are both extensions of the same sequence of divided powers, it follows that $y_{i+1}-y_{i}$ is a primitive. It is clear that the maximal coheight of the sum of two elements of different maximal coheight is the minimum of the two coheights. Therefore, we have an infinite set of primitives $\left\{y_{i+1}-y_{i}\right\}$, each one of the different maximal coheight. The set is independent, because, again, the maximal coheight of the sum of two elements of different maximal coheight is the minimum of the two coheights. This contradicts the assumption that $\operatorname{dim} P(H)<\infty$.

To see that $V \mid V^{\infty}(H) \rightarrow V^{\infty} \otimes k^{1 / p}$ is onto we first apply Remark 11 to $V^{\infty}(H) \otimes \bar{k}$. This remark implies that we can construct a basis of $V^{\infty}(H) \otimes \bar{k}$, such that each element is of the form ${ }^{n_{1}} l_{1} n_{2} l_{2} \ldots n_{m} l_{m}$, where each ${ }^{n_{i}} l_{i}$ is the $n_{i}$ th term of an infinite sequence of divided powers over ${ }^{1} l_{i}$, a member of a basis of $P\left(V^{\infty}(H) \otimes \bar{k}\right)$. (The above paragraph shows that every primitive in $V^{\infty}(H) \otimes k$ has coheight $\infty$ in $V^{\infty}(H) \otimes \bar{k}$. Therefore, every basis of $P\left(V^{\infty}(H) \otimes \bar{k}\right)$ is a Sweedler basis.) Since

$$
V\left({ }^{p n_{1}} l_{1}{ }^{p n_{2}} l_{2} \ldots p n_{m} l_{m}\right)={ }^{n_{1}} l_{1}{ }^{n_{2}} l_{2} \ldots n_{m} l_{m},
$$

we see that $V \mid V^{\infty}(H) \otimes \bar{k} \rightarrow V^{\infty}(H) \otimes \bar{k}$ is onto.

Now let $h \otimes \alpha \in V^{\infty}(H) \otimes k^{1 / p}$ and pick $\sum_{i} h_{i} \otimes \alpha_{i} \in V^{\infty}(H) \otimes \bar{k}$ such that $V\left(\sum_{i} h_{i} \otimes \alpha_{i}\right)=h \otimes \alpha$. Assume the $\alpha_{i}$ 's are independent over $k$ and $\alpha_{1} \in k$. Then $V\left(\sum_{i} h_{i} \otimes \alpha_{i}\right)=\sum_{i} V\left(h_{i}\right) \otimes \alpha_{i}^{1 / p}$ implies that $\sum_{i \neq 1} V\left(h_{i}\right) \otimes \alpha_{i}^{1 / p}=0$ (since $\left\{\alpha_{i}\right\}$ independent $/ k \Rightarrow\left\{\alpha_{i}^{1 / p}\right\}$ independent $\left./ k^{1 / p}\right)$. Therefore $V\left(h_{1} \otimes \alpha_{1}\right)=h \otimes \alpha \quad$ and $V \mid V^{\infty}(H) \rightarrow V^{\infty}(H) \otimes k^{1 / p}$ is onto.

Lemma 14. Assume $H$ has a Sweedler basis and let $H^{\prime}$ be the sub-Hopf algebra defined in Theorem 9. Consider $H$ as a subset of $H \otimes k^{1 / p}$. Then $H^{\prime} \subset k \circ V^{-1}(H)$.

Proof. If ${ }^{n} x$ is any element in any sequence of divided powers in $H$, then ${ }^{n} x \in k \circ V^{-1}(H)$ since $^{n} x \in V^{-1}\left({ }^{n / p} x \otimes 1\right)$ if $p \mid n$ and ${ }^{n} x \in V^{-1}(0)$ if $p \nmid n$ (Lemma 3). 
Theorem 4 implies that $H^{\prime}$ is generated as an algebra by elements of sequences of divided powers. Since $V$ preserves multiplication, $k \circ V^{-1}(H)$ is an algebra and $H^{\prime} \subset k \circ V^{-1}(H)$.

Corollary 15. $H$ and $H^{\prime}$ as in Lemma 14. Again, consider $H$ as a subset of $H \otimes k^{1 / p}$. Let $C$ be any subcoalgebra of $H$ contained in $k \circ V^{-1}(H)$. Then $C \subset H^{\prime}$.

Proof. Let $H^{\prime \prime}$ be the Hopf algebra generated by $C$. Since $V(H)$ is an algebra, $H^{\prime \prime} \subset k \circ V^{-1}(H)$. We shall show that $H^{\prime \prime} \subset H^{\prime}$.

$H^{\prime \prime} \subset k \circ V^{-1}(H)$ implies that for any $x \in H^{\prime \prime}$ and any $n, V^{n}$ applied to some linear multiple of $x$ will be in $H^{\prime \prime} \otimes k^{1 / p^{n}} \cap H=H^{\prime \prime}$. Therefore by Lemma 6, for any $n$, if $L_{n}$ is the set of primitives of coheight $n$ in $H^{\prime \prime}, L_{n} \otimes \bar{k}$ is the set of primitives of coheight $n$ in $H^{\prime \prime} \otimes \bar{k}$. Remark 12 says that $H^{\prime \prime} \otimes \bar{k}$ can be generated as an algebra by elements of sequences of divided powers. Since any primitive has the same coheight in $H^{\prime \prime}$ and $H^{\prime \prime} \otimes \bar{k}$, Theorem 4 tells us we could have picked these generating elements in $H^{\prime \prime}$. Consequently, $H^{\prime \prime}$ is generated by elements of sequences of divided powers, which implies by Theorem 9 that $H^{\prime \prime} \subset H^{\prime}$.

COROllary 16. $H$ and $H^{\prime}$ as in Lemma 14. Let $C$ be a coalgebra such that $C \approx \bigotimes_{\alpha} C_{\alpha}$ ( $\alpha$ tracing some index set, possibly infinite) with each $C_{\alpha}$ spanned by a single sequence of divided powers. Then, if $f: C \rightarrow H$ is a coalgebra map, $f(C) \subset H^{\prime}$.

Proof. As pointed out in the first sentence of the proof of Lemma 14, if $1,{ }^{1} l,{ }^{2} l, \ldots$ is a sequence of divided powers, ${ }^{i} l \in k \circ V^{-1}(H)$. Therefore, $\bigotimes_{\alpha}{ }^{i}{ }_{\alpha} l_{\alpha} \in V^{-1}\left({ }^{i}{ }^{i} p l_{\alpha} \otimes 1\right)$ if $p \mid i_{\alpha}$ for all $\alpha$ and $\bigotimes_{\alpha}{ }^{i}{ }_{\alpha} l_{\alpha} \in V^{-1}(0)$, otherwise. Since $V$ commutes with coalgebra maps [1, Proposition 4.1.5(a)] and since coalgebra maps preserve sequences of divided powers, $f(C) \subset k \circ V^{-1}(H)$. Therefore, by Corollary $15, f(C) \subset H^{\prime}$.

\section{BIBLIOGRAPHY}

1. R. Heyneman and M. E. Sweedler, Affine Hopf algebras. II, J. Algebra 16 (1970), 271-297.

2. K. Newman, Topics in the theory of irreducible Hopf algebras, Ph.D. Thesis, Cornell University, Ithaca, New York, 1970.

3. M. E. Sweedler, Hopf algebras, Math. Lecture Note Series, Benjamin, New York, 1969. MR 40 \#5705.

4. - - Hopf algebras with one grouplike element, Trans. Amer. Math. Soc. 127 (1967), 515-526. MR 35 \#1634.

5. —_, Weakening a theorem on divided powers, Trans. Amer. Math. Soc. 154 (1971), 427428.

McGill University, Montreal, Quebec, Canada 